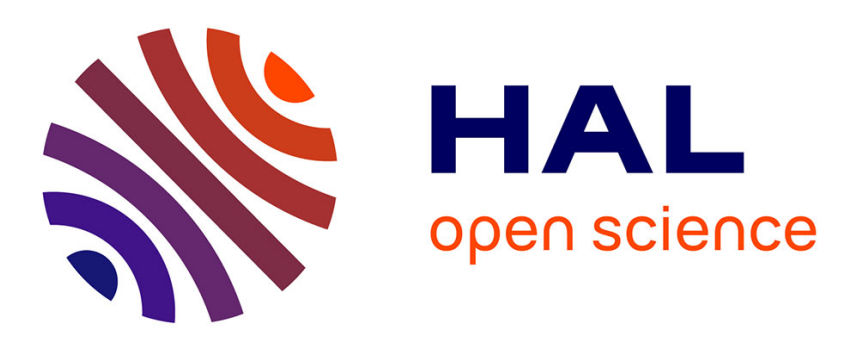

\title{
Confiance et reconnaissance envers les évaluateurs de Systèmes d'Information et Management (Editorial)
}

Cécile Godé, Jessie Pallud, François de Corbière

\section{To cite this version:}

Cécile Godé, Jessie Pallud, François de Corbière. Confiance et reconnaissance envers les évaluateurs de Systèmes d'Information et Management (Editorial). Systèmes d'Information et Management, 2020, 25 (1), pp.3. 10.3917/sim.201.0003 . hal-02791673

\section{HAL Id: hal-02791673 \\ https://hal.science/hal-02791673}

Submitted on 5 Jun 2020

HAL is a multi-disciplinary open access archive for the deposit and dissemination of scientific research documents, whether they are published or not. The documents may come from teaching and research institutions in France or abroad, or from public or private research centers.
L'archive ouverte pluridisciplinaire HAL, est destinée au dépôt et à la diffusion de documents scientifiques de niveau recherche, publiés ou non, émanant des établissements d'enseignement et de recherche français ou étrangers, des laboratoires publics ou privés. 


\title{
Éditorial
}

\section{CONFIANCE ET RECONNAISSANCE ENVERS LES ÉVALUATEURS DE SYSTEMMES D'INFORMATION ET MANAGEMENT}

\author{
Cécile Godé, Jessie Pallud, François de Corbière
}

ESKA | « Systèmes d'information \& management »

2020/1 Volume 25 | pages 3 à 5

ISSN 1260-4984

ISBN 9782747230407

Article disponible en ligne à l'adresse :

https://www.cairn.info/revue-systemes-d-information-etmanagement-2020-1-page-3.htm

Distribution électronique Cairn.info pour ESKA.

(C) ESKA. Tous droits réservés pour tous pays.

La reproduction ou représentation de cet article, notamment par photocopie, n'est autorisée que dans les limites des conditions générales d'utilisation du site ou, le cas échéant, des conditions générales de la licence souscrite par votre établissement. Toute autre reproduction ou représentation, en tout ou partie, sous quelque forme et de quelque manière que ce soit, est interdite sauf accord préalable et écrit de l'éditeur, en dehors des cas prévus par la législation en vigueur en France. Il est précisé que son stockage dans une base de données est également interdit. 


\title{
Éditorial
}

\section{Confiance et reconnaissance envers les évaluateurs de Systèmes d'Information et Management}

\author{
Cécile GODÉ, Jessie PALLUD et François DE CORBIÈRE
}

Parmi les multiples activités que nous menons de front en tant qu'enseignant-chercheur ou chercheur, la relecture des travaux de recherche des collègues en est une essentielle. En tant qu'expert du domaine, le relecteur est le garant de la rigueur, de la qualité et de la contribution scientifiques de l'article qui lui est soumis. En acceptant d'évaluer, il s'engage dans un processus long, fréquemment constitué de plusieurs allers-retours visant à améliorer et enrichir la recherche. Les rapports qu'il produit jouent un rôle clé dans la décision finale du rédacteur, qui les étudiera avec attention pour affiner son propre avis et choisir de refuser l'article ou de poursuivre le cycle d'amélioration.

La qualité scientifique de notre revue Systèmes d'Information et Management repose sur ce modèle d'évaluation par les pairs. Le processus se déroule en double aveugle : le rédacteur est le seul à connaître le nom des auteurs et des évaluateurs. Il orchestre ainsi une collaboration anonyme, temporaire et bénévole (ne l'oublions pas) visant un objectif commun : l'amélioration (si possible) et la validation scientifique de la recherche.
Ces dernières années, nombreux sont les débats et polémiques autour des vices et vertus du modèle d'évaluation de la recherche scientifique par les pairs. Parmi les nombreuses contributions à ce sujet, de virulentes critiques ont été publiées dans des revues prestigieuses telles que Journal of American Medical Association (Jefferson et al., 2002 ${ }^{1}$ ) ou encore Journal of the Royal Society of Medicine ${ }^{2}$ (Smith, 2006). Les auteurs y remettent en cause un processus considéré comme chronophage, peu objectif et dont l'efficacité scientifique ne serait pas démontrée.

En effet, et nous rejoignons en cela le constat déjà opéré par Régis Meissonnier à l'époque, «lorsque le processus d'évaluation par pairs n'est pas correctement balisé dans son esprit et sa forme, des dérives peuvent se développer " (2017, p. 3). Tout comme les rédacteurs en chef antérieurs, l'équipe actuelle est justement très attentive à construire et gérer un processus d'évaluation «balisé dans son esprit et sa forme ». Dans son esprit d'une part, car les évaluateurs sollicités sont à la fois experts dans le domaine et dignes de confiance. Il est ici question

\footnotetext{
${ }^{1}$ https://jamanetwork.com/journals/jama/article-abstract/194971

${ }^{2}$ https://www.ncbi.nlm.nih.gov/pmc/articles/PMC1420798/

$N^{\circ} 1-$ VOL. $25-2020$
} 
de compréhension et de bienveillance vis-à-vis de la recherche à évaluer. Dans sa forme d'autre part, car les évaluateurs produisent des commentaires qui doivent être pertinents et argumentés afin de favoriser une progression de la recherche ; ils sont en cela particulièrement attentifs à un certain nombre de points, notamment synthétisés par Bez et al. (2016) dans leur liste détaillée de critères à prendre en compte pour éviter un rejet aux auteurs. Des évaluateurs qui s'engagent également à respecter des échéanciers balisés, sur lesquels les marges de manœuvre sont étroites (de Corbière, 2019).

Pour toutes ces raisons, remerciements et gratitude à nos 79 évaluateurs 2019 !

\begin{tabular}{|c|c|c|}
\hline Anouck Adrot & François Deltour & Jean-François Lemoine \\
\hline Bénédicte Aldebert & Carine Dominguez-Péry & Katia Lobre \\
\hline Lise Arena & Aurélie Dudezert & Pierre Loup \\
\hline Saïd Assar & Karine Evrard-Samuel & Nada Maaninou \\
\hline Somsing Autcharaporn & Isabelle Fagnot & Rolande Marciniak \\
\hline Richard Baskerville & Samuel Fosso Wamba & Samuel Mercier \\
\hline Cédric Baudet & François Fulconis & Stéphanie Missonier \\
\hline Cécile Belmondo & Mike Gallivan & Lapo Mola \\
\hline Lamiae Benhayoun & Samia Gamoura & Chantal Morley \\
\hline Christine Bernadas & Lionel Garreau & Laure Muselli \\
\hline Vincent Berthelot & Bénédicte Geffroy & Roxana Ologeanu-Taddei \\
\hline Jean-François Berthevas & Corine Genet & Janosch Ortmann \\
\hline Marie Bia Figueiredo & Aurélie Girard & Gilles Paché \\
\hline Guillaume Biot-Paquerot & Mathias Guérineau & Amandine Pascal \\
\hline Sébastien Brion & Manon Ghislaine Guillemette & Alexandre Renaud \\
\hline Nabila Boukef & Véronique Guilloux & Sophie Renault \\
\hline Valérie Caraguel & Johanna Habib & Jean-Loup Richet \\
\hline Marie-Laurence Caron-Fasan & Joanne Hamet & Frantz Rowe \\
\hline Sabine Carton & Emilie Hoareau & Najma Saidani \\
\hline Kevin Carillo & Richard Huaman Ramirez & Caroline Sargis Roussel \\
\hline Antoine Chollet & Caroline Hussler & Alain Sone Mbassi \\
\hline Philippe Cohard & Hajer Kefi & Hirotoshi Takeda \\
\hline Alain Cucchi & Agnès Lancini & Mathieu Templier \\
\hline Nabyla Daidj & Marion Lauwers & Christina Tsoni \\
\hline Jean-François De Moya & Florence Laval & Claudio Vitari \\
\hline $\begin{array}{l}\text { Thibault } \\
\text { de Swarte }\end{array}$ & $\begin{array}{l}\text { Aurélie Leclercq- } \\
\text { Vandelannoitte }\end{array}$ & $\begin{array}{l}\text { Isabelle Walsh } \\
\text { Stefano Za }\end{array}$ \\
\hline
\end{tabular}


Dans ce numéro, trois articles sont présentés.

Le premier, d'Emmanuel Bertin, Aubin Colleaux et Aurélie Leclerc-Vandelannoitte, aborde la problématique de l'usage des outils de collaboration à l'ère du numérique. Ayant reçu le prix de la meilleure communication à la conférence de l'Association Information et Management en 2017, cet article analyse les processus d'articulation entre l'email, les réseaux sociaux d'entreprise et autres outils collaboratifs. À partir d'une étude empirique qualitative, les auteurs discutent des effets des outils collaboratifs sur la surcharge informationnelle et la surcharge collaborative. Enfin, ils mettent en exergue l'importance de la stratégie de transformation digitale des organisations qui doit accompagner la collaboration via les réseaux sociaux d'entreprise.

Le second article questionne la capacité d'absorption des entreprises, leur habilité à identifier des connaissances externes, les assimiler, les transformer et les exploiter à des fins de création de valeur. Khaireddine Mouakhar et Nordine Benkeltoum utilisent une méthode mixte de recherche pour analyser la relation entre la durée d'exposition à l'environnement et la capacité des entreprises de l'open source à créer de la valeur sur la base d'informations externes. Ils discutent ainsi de la corrélation entre la perception par les entreprises de leur intensité d'interaction avec l'environnement et la probabilité d'en exploiter les informations.

Enfin, le troisième article de ce numéro s'intéresse au crowdfunding, et plus particulièrement à l'equity crowdfunding.
Laurence Attuel-Mendes, Céline Soulas et Abdel Malik Ola questionnent ainsi le rôle du collectif incarné par la foule dans la prise de décision individuelle d'investissement sur les biotechnologies santé. Les auteurs combinent une démarche netnographique des campagnes de financement participatif lancées par des startups de biotechs et des entretiens auprès d'investisseurs. Ils montrent une sous-utilisation de l'information émise par la foule dans la fabrique de décision individuelle d'investissement, analysent le rôle joué par la foule dans cette décision et prolongent les travaux fondateurs sur la typologie des foules.

\section{RÉFÉRENCES CITÉES}

Bez, S.M., Berkowitz, H., Guérineau, M. (2016), «Coopérer pour publier: Une check-list collaborative pour éviter le desk reject », Le Libellio d'AEGIS, vol. 12, n² -Été 2016, Dossier Écrire et publier - p. 33-40.

de Corbière, F. (2019), « Du temps de la publication ", Systèmes d'Information et Management, vol. 24, n³, p. 3-5.

Jefferson T., Wager E. and Davidoff F. (2002), "Measuring the Quality of Editorial Peer Review", Journal of American Medical Association, vol. 287, n²1, p. 2786-2790.

Meissonier R. (2017), «Évaluer un article : quels syndromes éviter?", Systèmes d'Information et Management, vol. 22, n4, p. 3-8.

Smith R. (2006), "Peer review: A flawed process at the heart of science and journals", Journal of the Royal Society of Medicine, vol. 99, n4, p. 178-182. 